\title{
Atlantic air-sea interaction and model validation
}

\author{
Mark J. Rodwell and Chris K. Folland \\ Hadley Centre, Met Office, Bracknell, U.K.
}

\begin{abstract}
An analysis of observations from 1948-1998 suggests that the atmosphere in the North Atlantic region does respond to North Atlantic Sea-Surface Temperatures (SSTs) throughout the annual cycle. In the subtropics, high geopotential heights are seen to be a local response to warm SSTs. In winter, the North Atlantic Oscillation responds to a «tripole» pattern in North Atlantic SSTs. In summer, anticyclonicity over the U.K. is seen downstream of warm SST anomalies off Newfoundland and is possibly also related to warm subtropical SSTs. Such responses imply a degree of seasonal predictability and help quantify the strength of natural ocean-atmosphere coupled modes of variability. The average of an ensemble of 10 simulations of the HadAM3 atmospheric model forced with observed SSTs for the same period produces robust ocean-forced responses which agree well with those identified in the observations and with a previous model. The agreement is encouraging as it confirms the physical significance of the observational results and suggests that the model responds with the correct patterns to SST forcing. In the subtropics, the magnitude of the ensemble mean response is comparable with the observational response. In the extratropics, the magnitude of the model response is about half that of the observations. Although atmospheric internal variability may have affected the observed atmospheric patterns and there are considerations regarding the lack of two-way air-sea interaction with an atmospheric model, it is suggested that the model's extratropical response may be too weak. The 10 individual simulations of HadAM3 and 28 50-year periods of the ocean-atmosphere model, HadCM3, display similar results to each other with generally weaker ocean-forced links than observed. Seasonal predictability may, therefore, be too low in HadCM3 and low-frequency coupled modes under-represented. A moderate increase in the extratropics in the sensitivity of surface heat fluxes to surface temperatures is one possibility for improving these model deficiencies.
\end{abstract}

Key words North Atlantic - predictability - interaction - validation - NAO - anticyclonicity

\section{Introduction}

Observations and climate models display considerable low-frequency variability. A large part of this variability occurs naturally, due to air-sea interactions and ocean dynamics for example. To a first order approximation, such

Mailing address: Dr. Mark J. Rodwell, Hadley Centre for Climate Prediction and Research, Met Office, London Road, Bracknell, RG12 2SY, U.K.; e-mail: mark.rodwell@ metoffice.com variability may be considered to lead to lowfrequency oscillations of regional climate about any anthropogenically-forced climate trends. In reality, interactions will complicate this linear view. What is clear, however, is that climate models must be able to realistically represent the mechanisms involved in low-frequency variability if we are to improve our climate predictions and correctly attribute the reasons for past climate change.

Until recently, the climate of the North Atlantic region has generally been validated in models on relatively short timescales. Often the quantities examined are simply seasonal means of, for example, temperatures, zonal winds and transient eddy kinetic energy from rather short $(\sim$ 15-year) simulations. Such timescales are 
predominantly associated with atmospheric internal variability and there is little guarantee that the model is behaving well at longer (multiannual to multi-decadal) timescales when oceanatmosphere interactions become more important. Model validation at these longer timescales is urgently required. However, this is more difficult because the instrumental period is not sufficiently long to capture the full range of possible coupled variability.

Some of the mechanisms involved in lowfrequency variability operate at shorter timescales. Thus by trying to isolate short-timescale air-sea interaction in the observations, it may be possible to help validate modelled low-frequency variability. Here a diagnostic package, developed by Rodwell and Folland (2002) following work by Czaja and Frankignoul (1999), is used to focus on such short-timescale causal links. The package is based around the technique of maximal covariance analysis, based on Singular Value Decomposition (SVD), applied to Sea-Surface Temperatures (SSTs) and $500 \mathrm{hPa}$ geopotential height (Z500). By using SSTs that lead or lag the $\mathrm{Z} 500$ data, it is possible to gain an insight into causal links, even in the observational data. It is clear that this analysis can also be used to assess seasonal predictability.

Rodwell and Folland (2002), hereafter RF02, were able to demonstrate that the Hadley Centre's ocean-atmosphere climate model (HadCM3) represents reasonably well atmospheric internal variability and aspects of atmosphere-to-ocean forcing in the North Atlantic region. However, the model appeared to show a poor extratropical atmospheric response to SSTs. The conclusion was that coupled ocean-atmosphere modes, which may only be important on long timescales, could be absent or too weak in the model and, therefore, that low-frequency variability could be compromised. Any seasonal predictability to be gained from this coupling would also be lacking in the model. In their study, only four 50-year periods of the oceanatmosphere model were considered and only SSTs north of $10^{\circ} \mathrm{N}$ were used in the analysis. Here, results are presented of a major analysis of 28 50 -year periods of the ocean-atmosphere model control simulation with SSTs extending south to the equator. Instead of the 4 standard seasons used in RF02, 12 running 3-month seasons are used to gain a better understanding of the annual cycle of air-sea interaction.

RF02 also considered the Hadley Centre's atmospheric general circulation model (HadAM2b) in order to investigate directly results in Rodwell et al. (1999) involving one-way forcing from SSTs, and to address subsequent comments (e.g., Bretherton and Battisti, 2000). Here, a newer atmospheric model (HadAM3) is investigated, with 10 rather than 6 ensemble simulations. Importantly, HadAM3 is the atmospheric component of HadCM3 and so more direct comparisons can be made between the model results.

Section 2 describes the data and models. Section 3 explains the main method of analysis. Section 4 details the results of the analysis. Section 5 gives the conclusions of the study.

\section{Data and models}

We analyse gridded monthly mean $500 \mathrm{hPa}$ geopotential height (Z500), averaged into 12 running 3-month seasons and monthly-mean SSTs. The observations used cover the period 1948-1998. The Z500 data are from NCEP reanalyses (Kalnay et al., 1996) and the monthlymean SSTs are from HadISST (Rayner et al., 2002). We use the U.K. Met Office's HadAM3 Atmospheric General Circulation Model (AGCM) (Pope et al., 2000). Data comes from the period 1948-1998 of a ten-member ensemble of atmospheric simulations forced with the 'observed' HadISST SSTs and sea-ice extents. We also use a 1500-year control simulation of the U.K. Met Office's non-flux-adjusted Ocean-Atmosphere General Circulation Model (OAGCM), HadCM3 (Gordon et al., 2000). HadCM3 incorporates HadAM3 as its atmospheric component. All data are interpolated to the atmospheric model grid $\left(2.5^{\circ}\right.$ latitude $\times 3.75^{\circ}$ longitude).

\section{The analysis method}

The analysis method is the same as that used in RF02 with the exception that 12 running 3-month seasons (SON, OND, NDJ, etc.) are 
now considered rather than just the standard 4 3-month seasons. Although we recognise that teleconnections from other regions will play a part in Atlantic-region climate variability, we focus here on air-sea interactions in the North Atlantic region. Readers familiar with the method used in RF02 can skip to Section 4.

Briefly, a maximal covariance analysis, using the technique of Singular Value Decomposition (SVD) is applied to monthly-mean SSTs and seasonal-mean Z500. A lag is introduced so that, for example, August SSTs and SON Z500 are jointly analysed. The technique is called here «Lagged SVD» (LSVD). One aim of this method is to investigate causal links between the ocean and atmosphere with the limited observational data sets available and thus gain an insight into seasonal predictability. The second aim is to validate the air-sea links in our climate models by applying the same analysis to model output.

In an analogous way to EOF analysis, two fields $(\boldsymbol{S}(t)$, here SST, and $\boldsymbol{Z}(t)$, here Z500) are decomposed into a sum of spatially normalised patterns ( $\boldsymbol{p}_{k}$ and $\boldsymbol{q}_{k}$ ) multiplied by corresponding timeseries $\left(a_{k}(t)\right.$ and $\left.b_{k}(t)\right)$

$$
\boldsymbol{S}=\sum_{k} a_{k}(y) \boldsymbol{p}_{k} \quad \boldsymbol{Z}=\sum_{k} b_{k}(y) \boldsymbol{q}_{k}
$$

with $\boldsymbol{p}_{1}$ and $\boldsymbol{q}_{1}$ (the only patterns of interest here) chosen to maximise the covariance $\left\langle a_{1}, b_{1}\right\rangle \equiv \sigma_{1}$ under the constraints that $\boldsymbol{p}_{k} \cdot \boldsymbol{p}_{l}=\delta_{k l}, \boldsymbol{q}_{k} \cdot \boldsymbol{q}_{l}=\delta_{k l}$, $\left\langle a_{k}, b_{l}\right\rangle=\sigma_{k} \delta_{k l}$.

Of importance in this investigation is the first «covariance fraction», $C F \equiv \sigma_{1} / \sum_{k} \sigma_{k}$. The higher this fraction, the more dominant the first mode is at explaining joint variability. Further details of the SVD technique and a comparison with other similar techniques is given in Bretherton et al. (1992).

The SVD procedure will produce statistically covarying patterns even if there is no physical connection between the fields (i.e. no causal relationship). This is addressed by adopting the null hypothesis that there is no physical connection. If true, then temporally shuffling the sequences of the two fields (each shuffled differently) and applying the LSVD analysis should not lead to a statistically different outcome. Here, the combined action of shuffling and applying the LSVD is performed 100 times to produce a Monte Carlo-style test based on the value of the $\mathrm{CF}$. We calculate the percentage of test CFs that exceed the true $\mathrm{CF}$. This percentage is an estimate of the Significance Level (SL) of the strongest test that is satisfied. The smaller the SL, the stronger the test that is passed and the more confident one can be of a physical connection between the covarying patterns. Other possible test statistics include the first squared covariance (Czaja and Frankignoul, 1999) and the first squared covariance fraction (Iwasaka and Wallace, 1995). RF02 showed that the precise choice of test statistic does not affect the conclusions of the study. The method of shuffling is the same as that in Czaja and Frankignoul (1999) and RF02. Here the years of the two fields are randomly shuffled with the constraint that there must be a minimum separation of 2 years between the data from the two fields.

Further, we estimate the usefulness of any forecast system based on the LSVD technique applied to a single observed or modelled realisation. The procedure is similar to standard 'cross-validation' tests for other statisticallybased forecasting systems (Livezey, 1995). We take the original input data, $\boldsymbol{S}=\left\{\boldsymbol{S}_{i}\right\} ; \boldsymbol{Z}=\left\{\boldsymbol{Z}_{j}\right\}$ and remove one common year, $t$, to give $\left\{\boldsymbol{S}_{i \neq t}\right\}$, $\left\{\boldsymbol{Z}_{j \neq t}\right\}$. We apply the LSVD analysis to these reduced timeseries to obtain the pair of patterns $\boldsymbol{p}_{1}{ }^{(t)}, \boldsymbol{q}_{1}{ }^{(t)}$. 'Cross-validated timeseries' values are then defined by

$$
a_{1}^{\prime}(t)=S_{t} \cdot \boldsymbol{p}_{1}^{(t)} \quad b_{1}^{\prime}(t)=Z_{t} \cdot \boldsymbol{q}_{1}^{(t)}
$$

The cross-validated timeseries are built-up by repeating the procedure for each value of $t$. The cross-validated timeseries based on the leading field (SST) is referred to here as the "predicted timeseries'. The cross-validated timeseries based on the lagging (Z500) field is referred to here as the 'observed timeseries'. For display purposes, we normalise the timeseries so that they have a standard deviation of 1 and the illustrated patterns (which are from the full 50 years LSVD analysis) show the magnitude corresponding to 1 standard deviation in the timeseries. 
The correlation between the two crossvalidated timeseries

$$
\mathrm{COR} \equiv r_{a_{1}^{\prime}, b_{1}}
$$

is used to assess the skill of the forecast. Note that the cross-validated timeseries could be somewhat different from the non-cross validated timeseries, $a_{1}(t)$ and $b_{1}(t)$, and the correlation $r_{a_{1}, b_{1}}$, is found to be quite a bit larger than COR. This is no doubt an artifact of the covariance maximisation of the SVD technique and is the reason for crossvalidating when assessing true predictive skill.

Note that the patterns that emerge from an SVD analysis have an arbitrary joint sign so that the LSVD analysis could produce $-\left(\boldsymbol{p}_{1}^{(t)}, \boldsymbol{q}_{1}^{(t)}\right)$ rather than $\left(\boldsymbol{p}_{1}^{(t)}, \boldsymbol{q}_{1}^{(t)}\right)$. Here, the joint sign is made positive by ensuring that subtropical SST anomalies are positive.

\section{Results}

\subsection{SST-forced patterns}

Results from the LSVD analysis using the monthly mean SSTs that immediately precede each atmospheric seasonal field are shown in fig. 1a-o. The left and centre panels show the pattern and timeseries results from the observations whilst the right hand panels show the pattern results of the analysis applied to the mean of the 10 member AGCM ensemble. The aim of taking the average of 10 simulations is to significantly reduce the level of atmospheric internal variability and to allow a closer focus on the forced patterns. The analysis has been made for all 12 3-month running seasons but, for simplicity, only the standard seasons and JAS are shown. Comparison with RF02, who only analysed the standard seasons, reveals good agreement with their joint patterns and $\mathrm{CF}$ and SL values, despite our extension here of the SST region to the equator. Our CFs for the ensemble mean results are consistently higher than those of RF02 but this is consistent with the larger ensemble size in the present study.

The salient features of the results are:

i) Local subtropical links - With the exception of the observational results for DJF, all patterns from the observations and the model show direct links in the subtropics between (warm) SST anomalies and (high) geopotential heights.

ii) Tripole forces $N A O$ in winter and spring The model shows a clear tripole-like pattern of SST anomalies and an NAO-like Z500 response in DJF and MAM (fig. 1f,i). The high degree of atmospheric internal variability in winter appears to affect the significance of the observational patterns but the patterns themselves for DJF and MAM are in good agreement with those from the model. Correlations between global grid-point SST and the cross-validated SST timeseries (not shown) indicate that the North Atlantic SSTs are successfully isolated for DJF in the observational analysis. Weak ENSO-like SST anomalies are apparent in the model results.

Fig. 1a-o. Lagged Singular Value Decomposition (LSVD) analysis of monthly-mean Sea-Surface Temperature (SST) leading observed and simulated seasonal-mean $500 \mathrm{hPa}$ geopotential height (Z500) for the period 19481998. Left panels show the observational patterns of HadISST SST (coloured, in K) and NCEP Z500 (contour interval $5 \mathrm{~m}$, positive contours solid, negative contours dashed, zero contour dotted) for one standard deviation of the first LSVD mode. The «Covariance Fraction», CF, and the Significance Level (SL) of the CF for the mode is quoted in each title. Centre panels show normalised 'Observed' and 'Predicted' cross-validated timeseries for Z500 based on the observational analysis. The correlation between the two timeseries (COR) is given. The year corresponds to the first month of the Z500 data. The right-hand panels show the patterns derived from the ensemble mean of 10 simulations of the AGCM (HadAM3) for the same period. Results are shown for: a-c) August SST leading September -November (SON) Z500; d-f) November SST leading December - February (DJF) Z500; g-i) February SST leading March - May (MAM) Z500; j-1) May SST leading June - August (JJA) Z500, and m-o) June SST leading July - September Z500. 


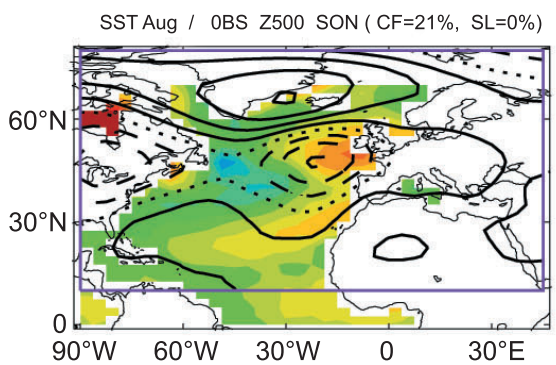

(a)

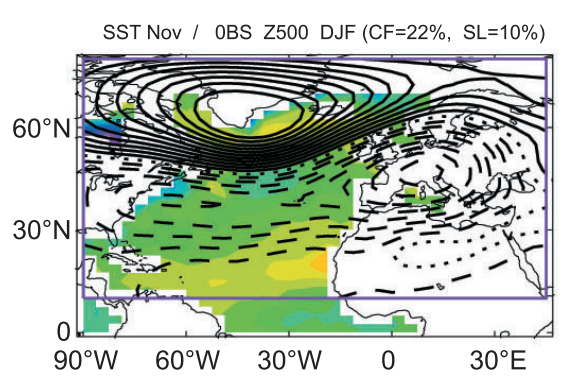

(d)

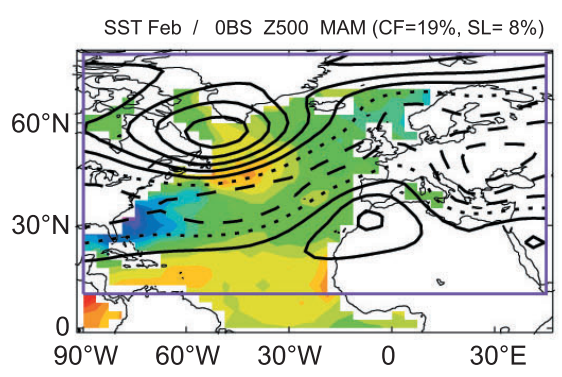

(9)

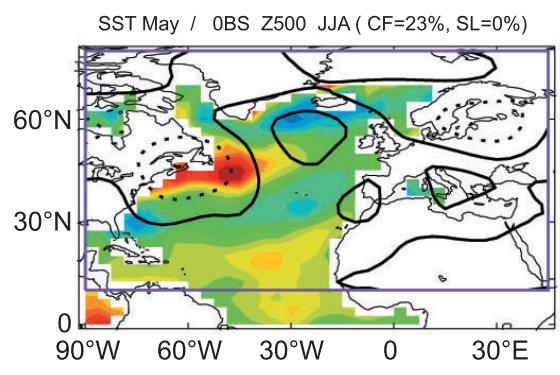

(j)

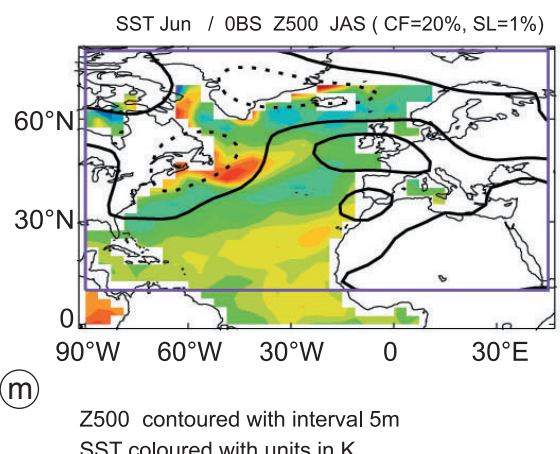

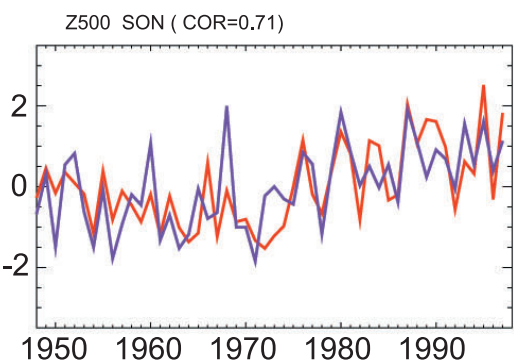

(b)

Z500 DJF ( COR=0.37)

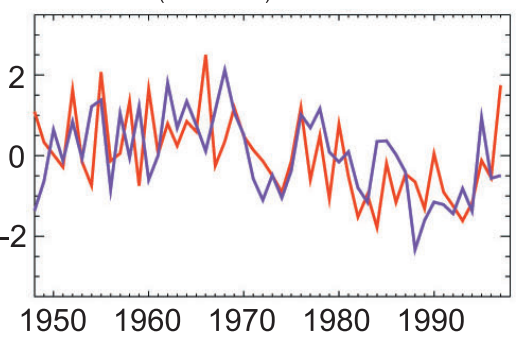

(e)

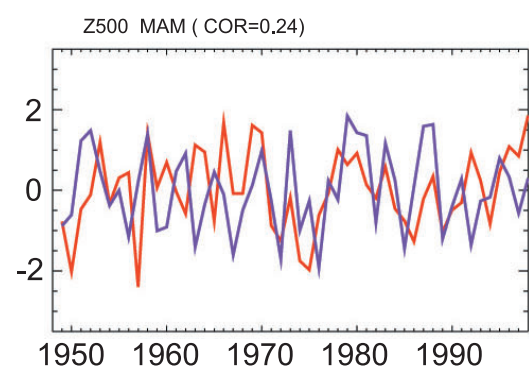

(h)

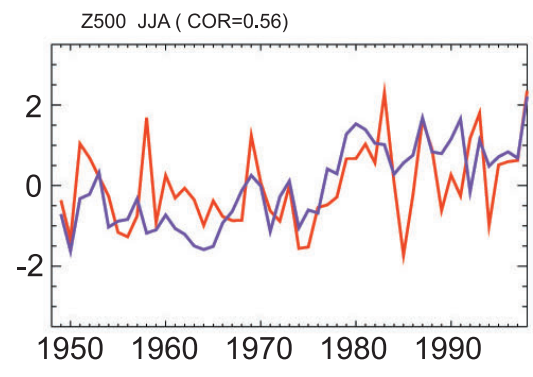

(k)

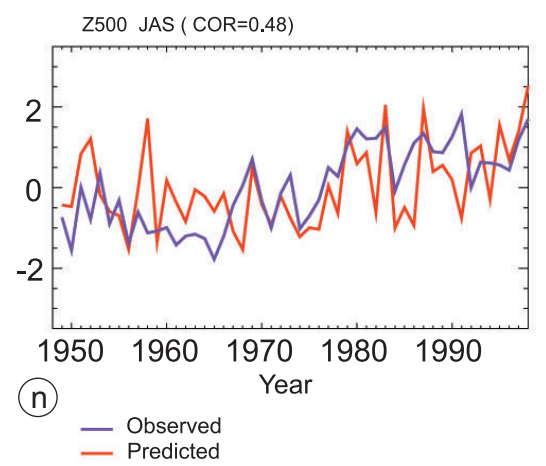

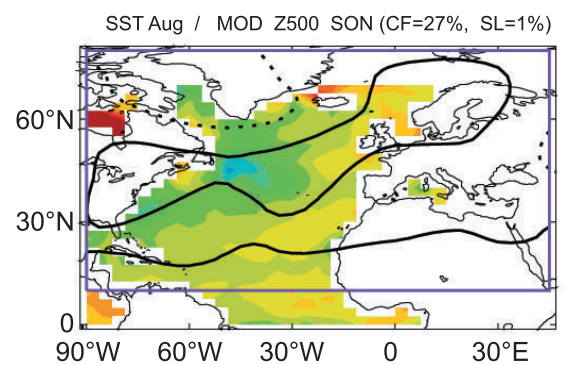

(C)

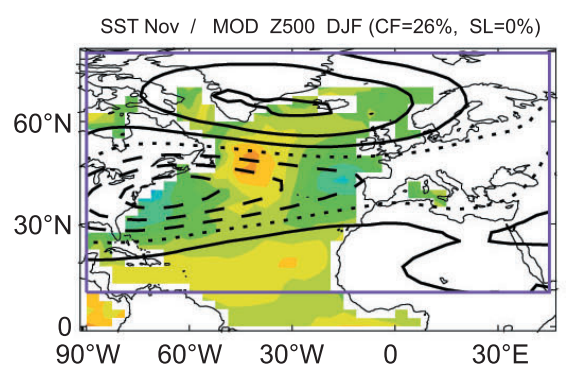

(f)

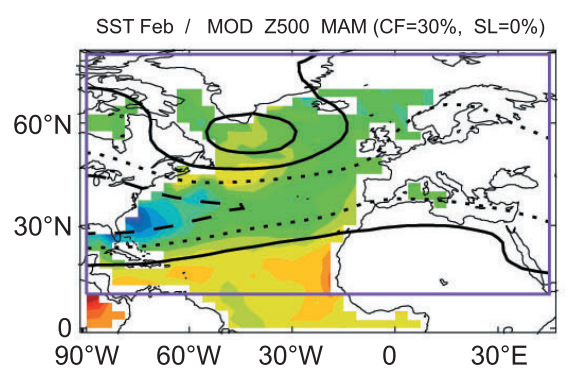

(i)

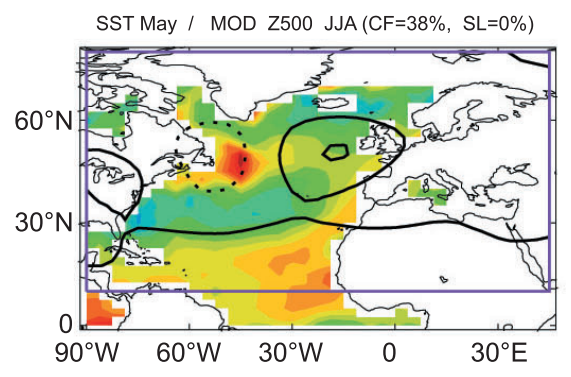

(I)

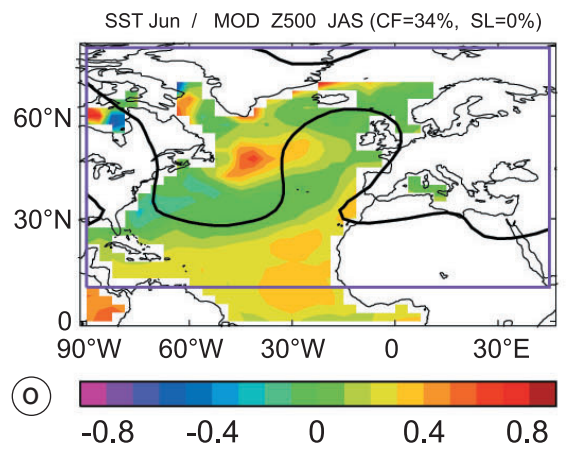


iii) U.K. summer anticyclonicity trend forced by SSTs - The observed summer anticyclonicity trend over the U.K. (Folland et al., 1988) is captured in the observational analysis (fig. $1 \mathrm{j}, \mathrm{m}$ ). The model patterns (fig. 11,o), which also show this feature, confirm quite conclusively that this is, at least partly, an ocean-forced signal. Although correlations show that ENSO-like SST anomalies have not been removed by the analysis in this season, the anticyclonicity is downstream (to the east) of warm SST anomalies and a (presumeably locally forced) cyclonic system off the Newfoundland coast. There is also an indication that the subtropical Atlantic SSTs may play a role in the extratropical response.

iv) Patterns not sensitive to trends - When the observed data are detrended prior to the analysis (not shown) the joint patterns remain essentially unchanged. The exception is for JFM, but neither the raw data nor the detrended data gave very significant patterns during this season.

v) Model and observational results agree With the exception of SON, there are strong extratropical similarities between the pairs of patterns obtained from the observations and the model. This agreement is encouraging in that it confirms the physical significance of the observational results and suggests that the model is responding reasonably well to SST forcing. The magnitudes of the Z500 patterns are similar for the observations and model ensemble mean in the subtropics. This is in agreement with the conclusion that at least $60 \%$ of AGCM seasonally averaged mean sea-level pressure variability is forced by SSTs in the tropics and subtropics (Rowell, 1998). Away from the tropics and subtropics, magnitudes of the model patterns become progressively weaker relative to those of the observations so that at about $50^{\circ} \mathrm{N}$, they have around half the magnitude of the observational patterns. This could be because the model being used here is an atmosphere-only model, with no feedback of the atmospheric signal onto the ocean. It may also be partly because atmospheric internal variability is 'contaminating' the observational patterns and partly because the atmospheric model's extratropical response to SSTs may be too weak. vi) Present model results agree with previous model - The patterns from the present model are remarkably similar to those obtained from the previous version of the model (HadAM2b) (fig. 8 in RF02). This robustness gives further confidence that the patterns do represent a true SST-forced signal. The agreement between models for the SON season is interesting because of the lack of agreement in the extratropics with the observational results for Z500. The suggestion is that either there is a systematic error that is robust across these two AGCMs or that internal variability in the observations is leading to a different (possibly still valid) extratropical link being highlighted.

vii) Seasonal predictability - Figure $1 \mathrm{~b}$ indicates a high degree of seasonal predictability for the Z500 pattern shown in fig. 1a for the SON season. One must be aware that sampling uncertainties could imply significant error bars on the predictive skill correlation value of $\mathrm{COR}=$ 0.71. RF02 found a correlation of 0.44 for this season. The present improvement could be due to the inclusion of tropical SSTs in the present study but is probably more a statement about the robustness of the correlation values themselves. Figure 1e,h,k,n indicates some seasonal predictability for the other seasons too although predictability for DJF and MAM (based on SSTs immediately prior to the season) is rather weak.

viii) Other seasons - For all seasons, the model ensemble mean patterns are highly significant (OND is the worst with $\mathrm{SL}=3 \%$ ). The year appears to be split between two pairs of patterns: the «north-south» (NAO) pattern seen in the cold half of the year NDJ to MAM (e.g., fig. 1f,i), and the «east-west» pattern seen in the warm half of the year MJJ to JAS (e.g., fig. 11,o). A transition from one set of patterns to the other is seen in the intervening seasons. In the observational results, the east-west pattern is robust from JJA to ASO (although significance drops for ASO: $\mathrm{SL}=22 \%$ ). The north-south pattern appears in NDJ, DJF and MAM but significance is lost for JFM and FMA, possibly due to the high level of atmospheric internal variability in winter but clearly there are several other plausible explanations. 


\subsection{Predictability at longer SST lead-times}

The observational analysis has been repeated for all SST lead-times up to 8 months before each season. The COR results are shown in the top left panel in fig. 2. The horizontal axis shows the 12 running seasons with the central month indicated. The vertical axis shows the SST lead-time from the central month. For example, the red $(\mathrm{O}, 2)$ square represents the high COR value shown in fig. 1b for August SST leading SON Z500. Crosses indicate configurations where the joint patterns are significant with SL $<10 \%$. We also reproduce the May/DJF COR result of RF02 with a different SST data set (HadISST instead of GISST) and with the SST region extended to the equator. The present May/NDJ result is slightly better than the May/DJF result with $\mathrm{COR}=0.48$, and $\mathrm{SL}=0 \%$. It was speculated in RF02, based on lagged correlations and observed surface latent heat fluxes, that this predictability, if real, may involve the preservation of thermal anomalies below the shallow summertime mixed layer and their re-emergence at the surface in the following winter. Long SST lead-time predictability is seen for summer seasons.

\subsection{Climate model validation}

The 52\% figure quoted in the top left panel of fig. 2 is the percentage of squares that represent configurations where the joint patterns are significant with SL $<10 \%$ (i.e. have crosses in). Although the degree of indepence of the squares is difficult to estimate, $52 \%$ is far more than the $10 \%$ one would expect by chance. To aid with model validation and to look for signs of multi-decadal variability in air-sea interaction, the same analysis has been applied to 2850 -year periods of the control simulation of the oceanatmosphere model. The results are shown in the remaining panels in fig. 2 (with the exception of the bottom right panel). It is clear that very little signal is seen. The mean percentage of squares representing significant patterns is $13 \%$, little more than expected by chance. The fact that no single 50-year period meets the $52 \%$ value of the observations is evidence for the statistical significance of the observational result. There is a possible indication of multi-decadal variability in coupling, hypothesised by RF02, with one panel showing $42 \%$ of patterns significant (particularly in summer) and some others showing only $2 \%$ but, since SST anomaly persistence and the running mean nature of the atmospheric seasons implies that the number of degrees of freedom is somewhat less than the number of squares, this variation may not be outside the range of reasonable chance. Crosses in the bottom right panel indicate where the observational COR values exceed all but at most 2 of the corresponding OAGCM correlations. This happens for $68 \%$ of the squares ( $43 \%$ of the observational COR values beat all the corresponding OAGCM correlations). The overwhelming conclusion is that the model does not capture an ocean-toatmosphere link as strongly as seen in the observations over the period 1948-1998.

One might ask whether there is an added «climate change» signal in the observations that boosts the perceived link. The best way to check this would be to investigate the behaviour of the OAGCM in the presence of historical anthropogenic forcing. However, the large number of anthropogenically-forced simulations required to be able to obtain a significant result have not been made. Even if they were made, there would still be concerns over the initialisation of the simulations owing to the lack of historical (ocean) data. Simple inspection of the trends in the NAO and June subtropical SSTs suggests that the observational record is not unusual and not outside the spread of model variations. The analysis of the detrended data (for SST leadtime of 2 months only) suggests only a modest decrease in COR values and this may be expected anyway because detrending will decrease the total variance in the data sets. The impact of a climate change signal present in the SSTs can be partially investigated using the AGCM results (see below).

Figure 3 shows all the COR values from the observations (filled circles), the OAGCM (plus signs) and AGCM (diagonal crosses) for SST leading Z500 by 2 months (e.g., Aug SST/SON Z500). Negative values imply no link is detected and, therefore, no predictability. It is clear that the observational COR values are generally higher than those for both models from SON to 


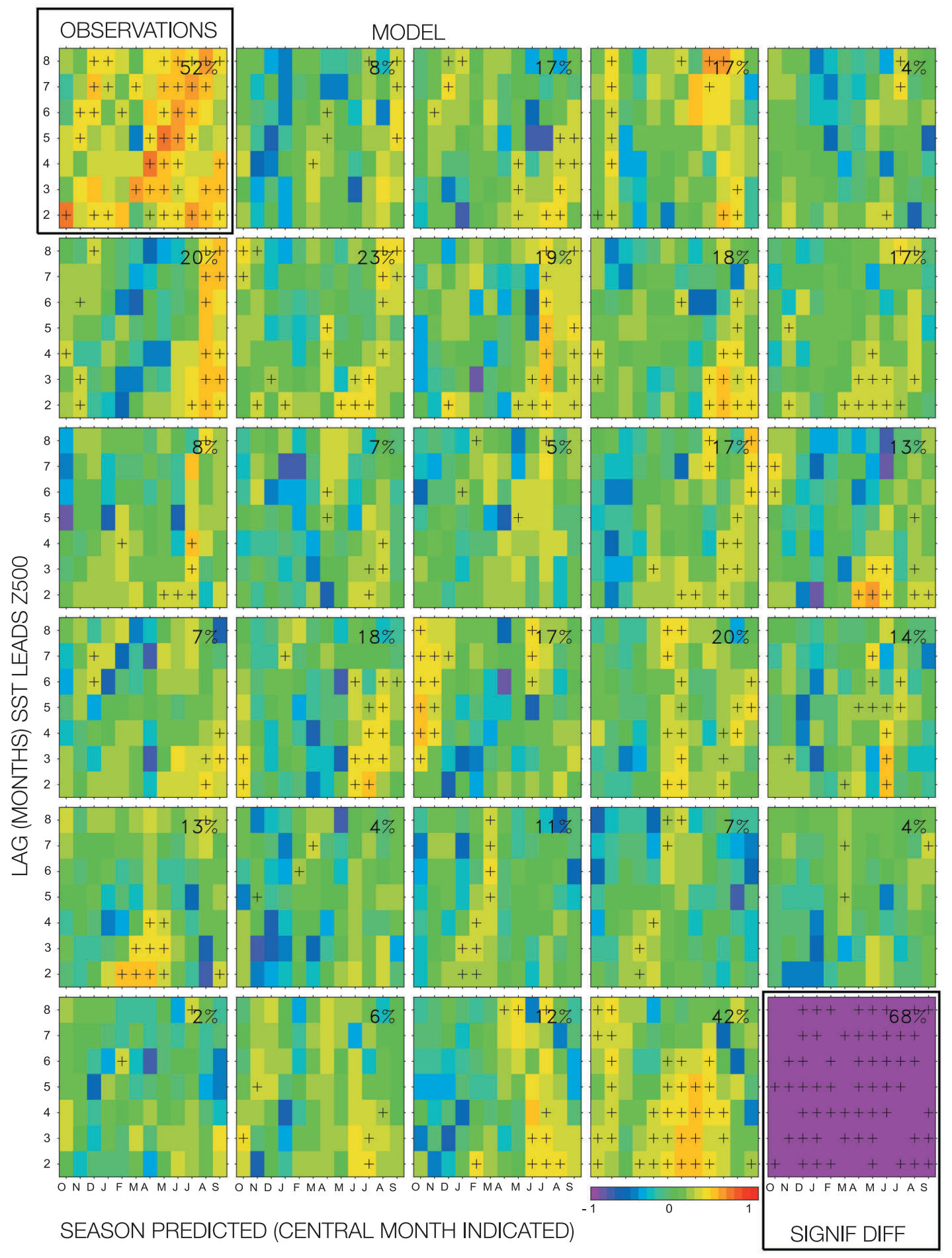

Fig. 2. Cross-validated correlation COR values (see Section 3 for definition) from the analysis of the observations (upper left panel) and 28 periods of the OAGCM (HadCM3) - remaining panels except lower right. COR values are given for all SST lead-times up to 8 months. The months on the $x$-axis indicate the central month of the atmospheric season being predicted. For example, square D7 indicates May SST leading NDJ Z500. Plus signs indicate SST and Z500 configurations where the patterns are significant at the $10 \%$ level (i.e. SL $<10 \%$ ). The percentages indicate the percentage of squares for which SL $<10 \%$. The lower right panel indicates the configurations for which the observational COR values exceed all but at most two of the 28 model COR values. This occurs for 68\% of all squares (as indicated). 


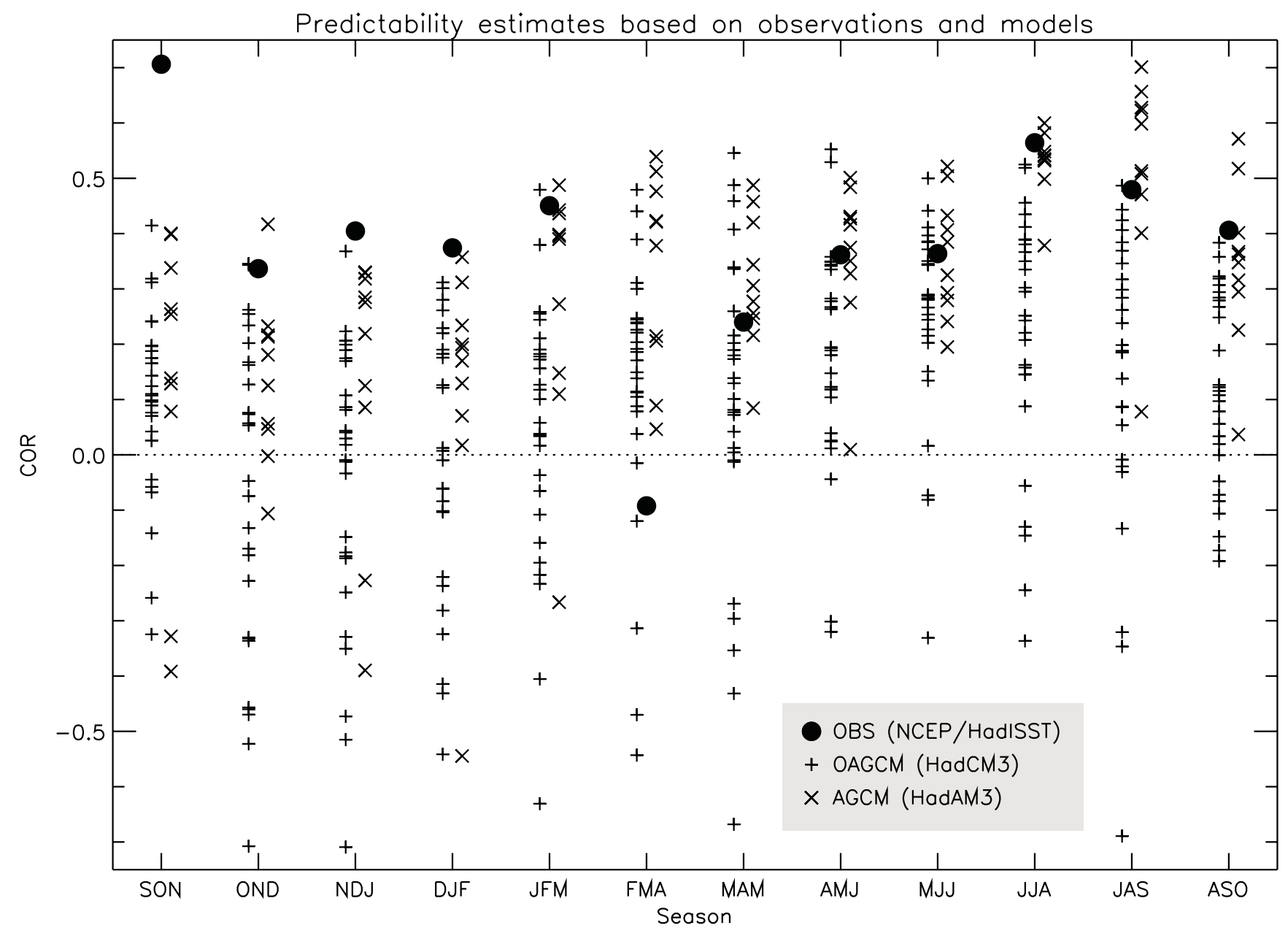

Fig. 3. Cross-validated correlations (CORs) obtained from the observations (circles), OAGCM (plus signs) and AGCM (diagonal cross crosses) when SSTs lead Z500 by two months.

JFM (cold season). From AMJ to ASO (warm season), the observations are generally within both model COR ranges but above average. This is particularly true when compared with the OAGCM results JJA-ASO. The observed COR value for FMA is negative and exceeded on average by both models. The reason for the negative observational COR value (and for a poor CF significance level of SL $=40 \%$ ) appears to be because the first two LSVD modes for this season are not well distinguished, with $\mathrm{CF}$ $($ mode 1$)=19 \%$ and $\mathrm{CF}(\operatorname{mode} 2)=17 \%$. Whether there really are two distinct physical modes or whether internal variability is dominating the analysis in this season is unclear at present. The conclusion is that the biggest potential for model improvement is likely to be in the autumn and winter seasons. It is interesting to note that the AGCM COR values tend to occupy the upper ranges of the OAGCM COR values. This may suggest that the recent observed period has involved stronger air-sea interaction than previous periods (as suggested by RF02) or it might partly reflect a climate change signal in the observed SSTs. It may simply reflect differences in the design of AGCM and OAGCM simulations.

\section{Summary}

A lagged singular value decomposition technique has been applied to observations from 1948 to 1998 , 10 simulations for the same period of an atmospheric model (HadAM3) and 28 50-year periods of an ocean-atmosphere 
model (HadCM3) simulation in order to validate mechanisms of low-frequency variability. Model and observational results suggest that:

- The atmosphere in the North Atlantic region does respond to North Atlantic Sea-Surface Temperatures (SSTs) throughout the annual cycle.

- High (low) subtropical geopotential heights are seen to be a local response to warm (cool) SSTs.

- The winter North Atlantic Oscillation responds to a «tripole» pattern in North Atlantic SSTs.

- Summer anticyclonicity over the U.K. is seen down-stream of warm SST anomalies off Newfoundland and is possibly also related to warm subtropical North Atlantic SSTs or SSTs elsewhere.

- In the subtropics, the magnitude of the atmospheric model ensemble mean response is comparable with the observational response. In the extratropics, the magnitude of the model response is about half that of the observations. Along with other possible reasons, it is suggested that the model's extratropical response to SSTs may be too weak.

- Analysis of the 10 individual simulations of atmospheric model and the 2850 -year periods of the ocean-atmosphere model tend to confirm that the modelled atmosphere responds too weakly to North Atlantic SST forcing, particularly in autumn and winter.

The suggestion from this work is that the atmospheric model does respond with the correct patterns to SST forcing although this may be too weak in the extratropics in autumn and winter. It is recommended that the sensitivity of extratropical heat-fluxes to surface temperatures is investigated, with the anticipation that an increase in sensitivity may improve modelled air-sea interactions.

\section{Acknowledgements}

The authors would like to thank David Parker and Jeff Knight for useful discussions in connection with this paper. This work was funded jointly by the EC SINTEX project and the U.K. Government Meteorological Research Programme.

\section{REFERENCES}

Bretherton, C.S. and D.S. Battisti (2000): An interpretation of the results from atmospheric general circulation models forced by the time history of the observed sea surface temperature distribution, Geophys. Res. Lett., 27 (6), 767-770.

Bretherton, C.S., C. SMith and J.M. WALlaCE (1992): An intercomparison of methods for finding coupled patterns in climate data, J. Climate, 5, 541-560.

CZAJA, A. and C. FRANKIGNOUL (1999): Influence of North Atlantic SST on the atmospheric circulation, Geophys. Res. Lett., 26 (19), 2969-2972.

Folland, C., D. PARKER, M. WARD and C. COLMAN (1988): Sahel rainfall, N Hemisphere circulation anomalies and worldwide sea temperature changes, in Persistent Meteo-Oceanographic Anomalies and Teleconnections, Pontificiae Academiae Scientiarum Scripta Varia, 69, 393-436.

Gordon, C., C. CoOper, C.A. Senior, H. Banks, J.M. GREGORY, T.C. JOHNS, J.F.B. MitCHELL and R.A. WoOD (2000): The simulation of SST, sea ice extents and ocean heat transports in a version of the Hadley Centre coupled model without adjustments, Climate Dyn., 16, 147-168.

IWASAKA, N. and J.M. WALLACE (1995): Large scale air sea interaction in the Northern Hemisphere from a view point of variations of surface heat by SVD analysis, $J$. Meteorol. Soc. Japan, 73 (4), 781-794.

Kalnay, E., M. Kanamitsu, R. Kistler, W. Collins, D. DeAVen, L. Gandin, M. Iredell, S. SAHA, G. White, J. Woollen, Y. Zhu, M. Chelliah, W. EbisuZaki, W. Higgins, J. JANOWIAK, K.C. MO, C. ROPELEWSKI, J. WANG, A. LEETMAAN, R. REYNOLDS, R. JENNE and D. JOSEPH (1996): The NCEP/NCAR 40-year reanalysis project, Bull. Am. Meteorol. Soc., 77 (3), 437-471.

LIVEZEY, R.E. (1995): The evaluation of forecasts, in Analysis of Climate Variability, edited by H. VON STORCH and A. NAVARRA (Springer, Berlin), p. 334.

Pope, V.D., M.L. GAllani, P.R. Rowntree and R.A. STRATTON (2000): The impact of new physical parametrizations in the Hadley Centre climate modelHadAM3, Climate Dyn., 16, 123-146.

RAyner, N.A., D.E. PARKer, C.K. Folland, L.V. ALEXANDER, E.B. HORTON and D.P. ROWELl (2002): Globally complete analyses of sea-surface temperature, sea-ice and marine air temperature,1871-2000, J. Geophys. Res. (manuscript in preparation).

RoDWELL, M.J. and C.K. FOLLAND (2002): Atlantic air-sea interaction and seasonal predictability, Q.J.R. Meteorol. Soc., 128, 1413-1443.

RodwEll, M.J., D.P. RowEll and C.K. FollAND (1999): Oceanic forcing of the winter North Atlantic Oscillation and European climate, Nature, 398, 320-323.

RowelL, D.P. (1998): Assessing potential seasonal predictability with an ensemble of multidecadal GCM simulations, J. Climate, 11, 109-120. 